\title{
Optically active quantum dots in monolayer $\mathrm{WSe}_{2}$
}

\author{
Ajit Srivastava ${ }^{1 \star \dagger}$, Meinrad Sidler"i, Adrien V. Allain ${ }^{2}$, Dominik S. Lembke ${ }^{2}$, Andras Kis ${ }^{2}$ \\ and A. Imamoğlu ${ }^{1 \star}$
}

\begin{abstract}
Semiconductor quantum dots have emerged as promising candidates for the implementation of quantum information processing, because they allow for a quantum interface between stationary spin qubits and propagating single photons ${ }^{1-3}$. In the meantime, transition-metal dichalcogenide monolayers have moved to the forefront of solid-state research due to their unique band structure featuring a large bandgap with degenerate valleys and non-zero Berry curvature 4 . Here, we report the observation of zero-dimensional anharmonic quantum emitters, which we refer to as quantum dots, in monolayer tungsten diselenide, with an energy that is $20-100 \mathrm{meV}$ lower than that of two-dimensional excitons. Photon antibunching in second-order photon correlations unequivocally demonstrates the zero-dimensional anharmonic nature of these quantum emitters. The strong anisotropic magnetic response of the spatially localized emission peaks strongly indicates that radiative recombination stems from localized excitons that inherit their electronic properties from the host transition-metal dichalcogenide. The large $\sim 1 \mathrm{meV}$ zero-field splitting shows that the quantum dots have singlet ground states and an anisotropic confinement that is most probably induced by impurities or defects. The possibility of achieving electrical control in van der Waals heterostructures ${ }^{5}$ and to exploit the spin-valley degree of freedom 6 renders transitionmetal-dichalcogenide quantum dots interesting for quantum information processing.
\end{abstract}

Advances in semiconductor-based quantum information processing have been made on two disjoint fronts. While optically active self-assembled quantum dots with deep electron and hole confinement allow for the realization of highly efficient single-photon sources $^{7}$, all-optical manipulation of confined spins ${ }^{8,9}$ and a spinphoton quantum interface ${ }^{3,10}$, the random nature of their growth seems to be the biggest hindrance to their use in scalable quantum information processing. In contrast, electrically defined single $^{11}$ or double quantum dots ${ }^{12}$ hosting one or two excess electrons have been shown to exhibit long spin coherence times together with a clear path towards integrated scalable devices. However, weaker confinement has precluded the possibility to reliably transfer quantum information from spins to photons in these systems. Quantum dots in monolayer transition-metal dichalcogenides (TMDs) have the potential to combine the desirable features of both optically active and electrically defined quantum dots. Although we report tungsten diselenide $\left(\mathrm{WSe}_{2}\right)$ quantum dots that appear due to uncontrolled impurity- or defect-induced traps, the two-dimensional nature of these materials makes it easier to electrically control the local potentials on a scale of a few tens of nanometres. More importantly, strong electron-hole binding in TMDs suggests that it would be possible to obtain a quantized optical excitation spectrum due to trapping of excitons or trions in large electric field gradients induced by external gates ${ }^{13}$
The samples we studied were obtained by mechanical exfoliation of $\mathrm{WSe}_{2}$ synthetic crystals onto heavily doped silicon substrates with a $285 \mathrm{~nm} \mathrm{SiO} \mathrm{S}_{2}$ layer on top (see Methods). Figure 1a (left) presents photoluminescence spectra of a monolayer flake (flake 0 ) of $\mathrm{WSe}_{2}$. These spectra do not show sharp emission peaks at either of two different excitation laser powers $\left(P_{\text {exc }}\right)$. The spectra clearly show two high energy peaks: exciton $\left(\mathrm{X}^{0}\right)$ emission at $708 \mathrm{~nm}$ and charged exciton (trion) emission $\left(\mathrm{X}^{-}\right)$at around $722 \mathrm{~nm}$ at high $P_{\text {exc }}$. These assignments are in accordance with previous reports of photoluminescence from monolayer $\mathrm{WSe}_{2}$ (ref. 14). The third feature of the spectra is the broad emission to the lower energy side of the trion peak, which has been attributed previously to impurity/defect-trapped excitons in $\mathrm{WSe}_{2}$ and other TMDs ${ }^{15}$. At low $P_{\text {exc }}$, the $\mathrm{X}^{0}$ and $\mathrm{X}^{-}$peaks are too weak to be detected although the impurity band peak can still be seen, suggesting a starkly different power dependence of the impurity band compared to the $\mathrm{X}^{0}$ and $\mathrm{X}^{-}$peaks. The detailed power dependence of $\mathrm{X}^{0}, \mathrm{X}^{-}$and the impurity band presented in Fig. 1a (right) confirms this claim; the integrated photoluminescence intensity of the $\mathrm{X}^{0}$ and $\mathrm{X}^{-}$peaks shows a linear dependence up to the highest $P_{\text {exc }}(\sim 320 \mu \mathrm{W})$, but that of the impurity band exhibits saturation behaviour (sublinear dependence), even at powers as low as $\sim 100 \mathrm{nW}$. We do not observe any broadening of the $\mathrm{X}^{0}$ and $\mathrm{X}^{-}$peaks, even at the highest $P_{\text {exc }}$, whereas the spectral features of the impurity band change significantly with incident laser power (Supplementary Fig. 1).

This type of saturation behaviour at relatively low powers compared to free excitons is consistent with the photoluminescence that stems from defects and impurities, which act as two-level emitters. It is well known from optical investigations of quantum well structures in III-V semiconductors that in low-quality samples with high defect or impurity density, the photoluminescence is dominated by redshifted emission from these localized states. This is a consequence of the fact that optically generated excitons relax by phonon emission into the localized states, quenching the delocalized exciton emission.

Figure $1 \mathrm{~b}$ presents photoluminescence spectra for another $\mathrm{WSe}_{2}$ flake (flake 1), in which the impurity band is composed of sharp emission peaks that become prominent at lower $P_{\text {exc }}$. For $P_{\text {exc }} \leq 1 \mu \mathrm{W}$, only sharp peaks are visible and the $\mathrm{X}^{0}$ peak becomes too weak to be detected. Figure 1c (right) shows a spatial map of integrated photoluminescence intensity for $\mathrm{X}^{0}$ (area enclosed by the black contour) and two different sharp emission peaks (Fig. 1c, left) on flake 1 obtained by raster-scanning the sample with piezoelectric positioners. The sharp emission peaks are seen to be spatially localized such that photoluminescence spectra at energies lower than the $\mathrm{X}^{-}$emission appear strikingly different at different locations on the flake. We observed more than 20 sharp emission peaks in an area of $\sim 8 \mu^{2}$ (corresponding to a density of more than 2.5 peaks per $\mu \mathrm{m}^{2}$ ). High-resolution photoluminescence spectra of the two sharp peaks (labelled QD1F1 and QD2F1) were obtained with $P_{\mathrm{exc}}<1 \mu \mathrm{W}$ and exhibit extremely 

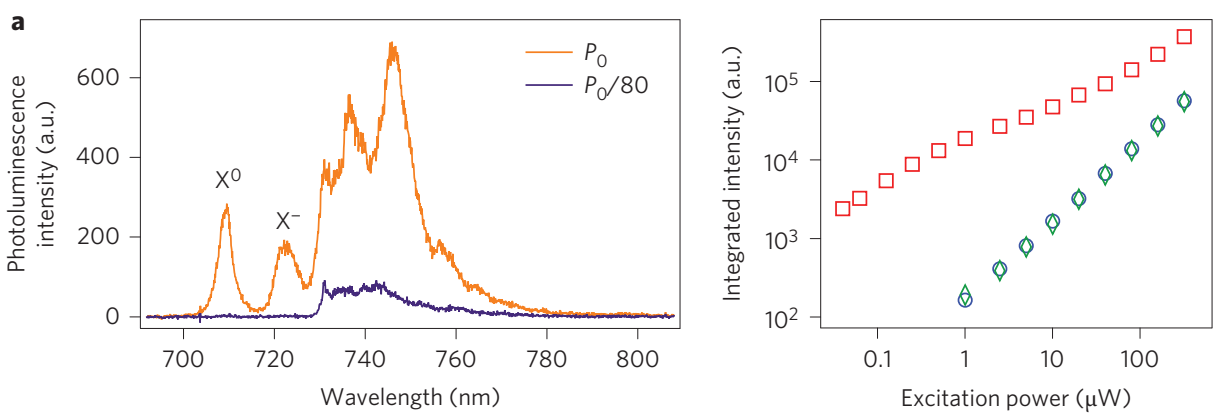

b
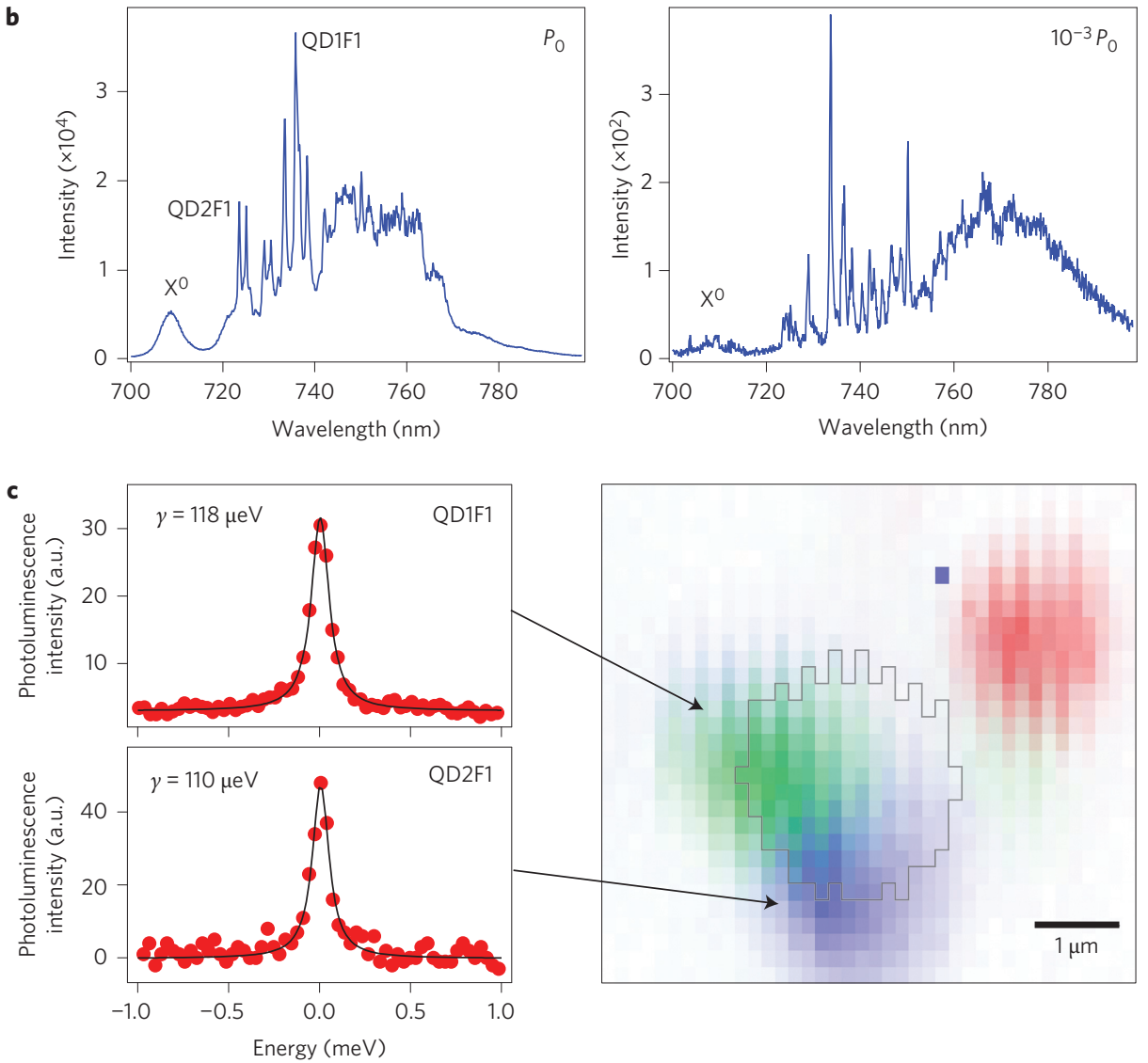

Figure 1 | Photoluminescence of monolayer $\mathbf{W S e}_{2}$ flakes. a, Left: Low-temperature ( $4.2 \mathrm{~K}$ ) photoluminescence spectra of a monolayer flake (flake 0 ), which do not show sharp peaks in emission at two different powers of the incident laser (HeNe, $632.8 \mathrm{~nm}$ ). Vastly different power dependences are observed for the exciton $\left(\mathrm{X}^{0}\right)$, trion $\left(\mathrm{X}^{-}\right)$peaks and the broad feature (impurity band) at longer wavelengths. Right: Detailed laser power dependence, showing linear behaviour of the integrated photoluminescence intensity for $X^{0}$ (blue circles) and $X^{-}$(green diamonds) peaks, but a sublinear dependence for the impurity band photoluminescence (red squares). b, Low-temperature photoluminescence spectra of a monolayer flake (flake 1) showing sharp emission lines at high and low laser power (left and right panels, respectively). The $X^{0}$ peak is too weak to be detected at low power, but the sharp emission peaks show saturation behaviour similar to the impurity band photoluminescence in $\mathbf{a}$. c, Right: Spatial map of photoluminescence from flake 1 showing localized emission of the sharp peaks. Green (blue) region: spatial extent of the photoluminescence peak labelled QD1F1 (QD2F1) in relation to the region where the $X^{0}$ photoluminescence reduces to half its maximum value (area enclosed by the grey contour). Red region: spatial extent of the sharp peak located close to the edge of the flake, which has reduced overlap with the $X^{0}$ region. Left: High-resolution photoluminescence spectra of QD1F1 and QD1F2, showing extremely narrow linewidths.

narrow linewidths of less than $120 \mu \mathrm{eV}$, possibly limited by spectral diffusion of the emission peak as discussed below. We rule out Raman scattering as the origin of the sharp features because changing the energy of the incident laser does not result in any corresponding shift in the emission energy. It is interesting to note that we observe strong photoluminescence from a region (coloured red in Fig. 1c, right) where the exciton emission is more than an order of magnitude weaker than its maximum value. We also measured the differential reflectance of the flake using a broadband white-light source, which shows a clear peak only for $\mathrm{X}^{0}$ (ref. 16).

To prove that the sharp emission peaks originate from localized excitons with an anharmonic spectrum, we measured the photon correlation function $g^{2}(\tau)$ using a Hanbury-Brown-Twiss (HBT) set-up with two single photon-counting avalanche photodiodes (APDs). Because $g^{2}(\tau=0)$ gives the likelihood for detecting two photons simultaneously, an ideal single zero-dimensional emitter 
a
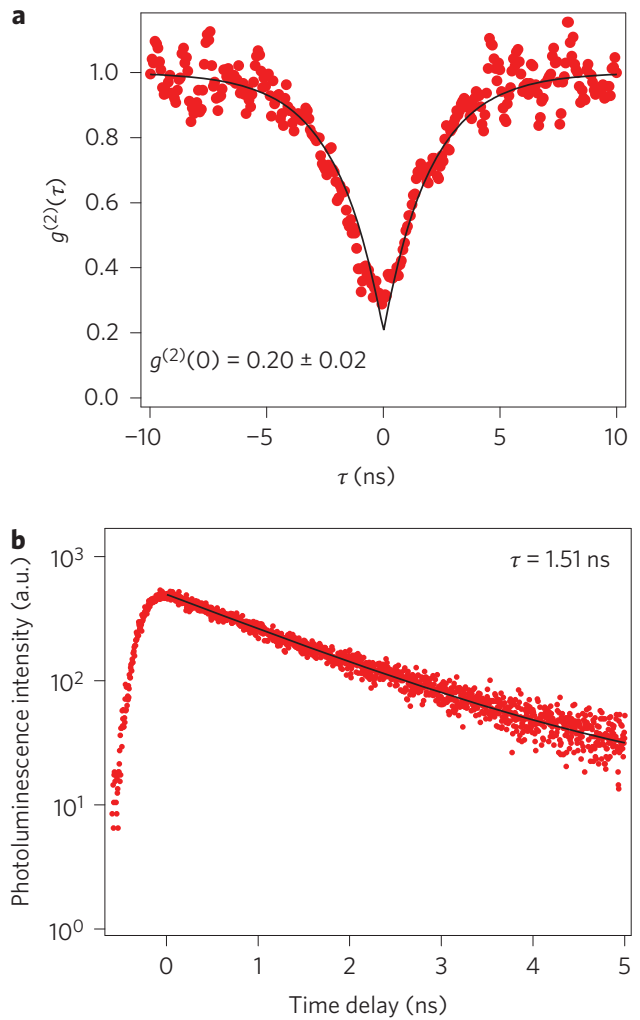
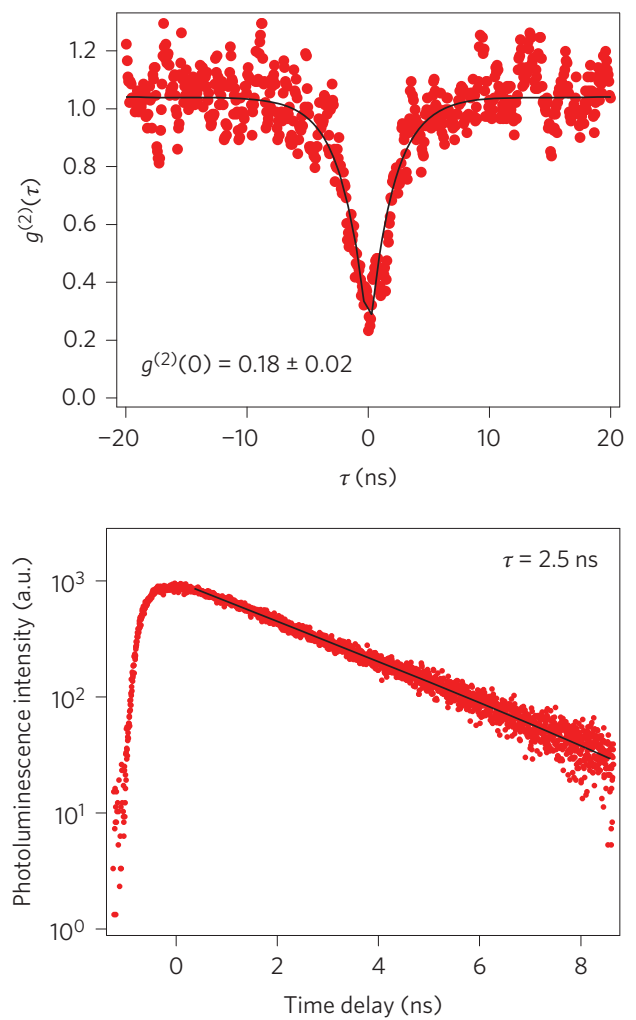

Figure 2 | Photon correlations and photoluminescence lifetimes of quantum dots. a, Second-order photon correlation function, $g^{2}(\tau)$, of photoluminescence measured using an HBT set-up, showing a pronounced dip (antibunching) at zero time delay for emission lines QD1F1 (left) and QD4F1 (right), confirming that they originate from zero-dimensional emitters-quantum dots. b. Time-resolved photoluminescence of QD1F1 (left) and QD3F1 (right), measured using a pulsed Ti:sapphire laser ( $5 \mathrm{ps})$, showing the long lifetime of the excited state typical of zero-dimensional emitters.
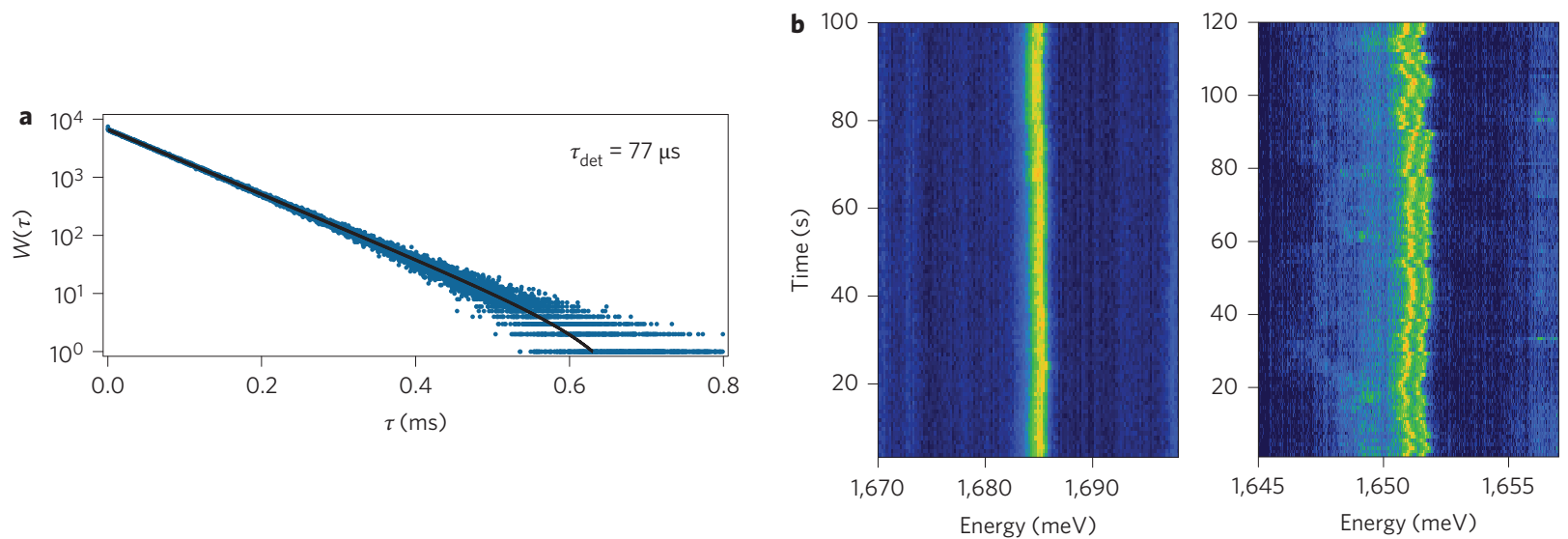

Figure 3 | Stability of emission and spectral wandering of quantum dots. a, Waiting time distribution of photoluminescence from QD1F1 as a function of time delay between successive single-photon detection events, showing single exponential decay, indicating the absence of intensity intermittency on timescales larger than the average time for photon detection, $\tau_{\text {det. }} \mathbf{b}$, Left: Time trace of photoluminescence emission from a typical quantum dot measured with low spectral resolution, showing stable peak position. Right: High-resolution photoluminescence time trace of another quantum dot showing spectral wandering with a range of $1 \mathrm{meV}$. The synchronized wandering of the two peaks strongly suggests that they arise from the same quantum dot and are associated with electron-hole exchange split exciton transitions.

with uncorrelated background contribution gives $g^{2}(0)=0$. In general, a measured value of $g^{2}(0)<0.5$ unequivocally proves that the source of emission originates predominantly from a single anharmonic emitter-a quantum dot. Figure 2a presents the $g^{2}(\tau)$ measurement results for QD1F1 and QD4F1, which yield $g^{2}(0)=0.20 \pm 0.02$ and $g^{2}(0)=0.18 \pm 0.02$, respectively. We therefore conclude that the sharp peaks are indeed associated with quantum dot photoluminescence. Figure $2 \mathrm{~b}$ shows the photoluminescence lifetime of quantum dots QD1F1 and QD3F1 on flake 1, which was measured by excitation with a $\sim 5$ ps pulsed Ti:sapphire laser tuned into resonance with the $\mathrm{X}^{0}$ transition and then sending the spectrally filtered output around the quantum dot wavelength to a single-photon-counting APD. The measured long lifetime of few nanoseconds is consistent with behaviour shown by 
a

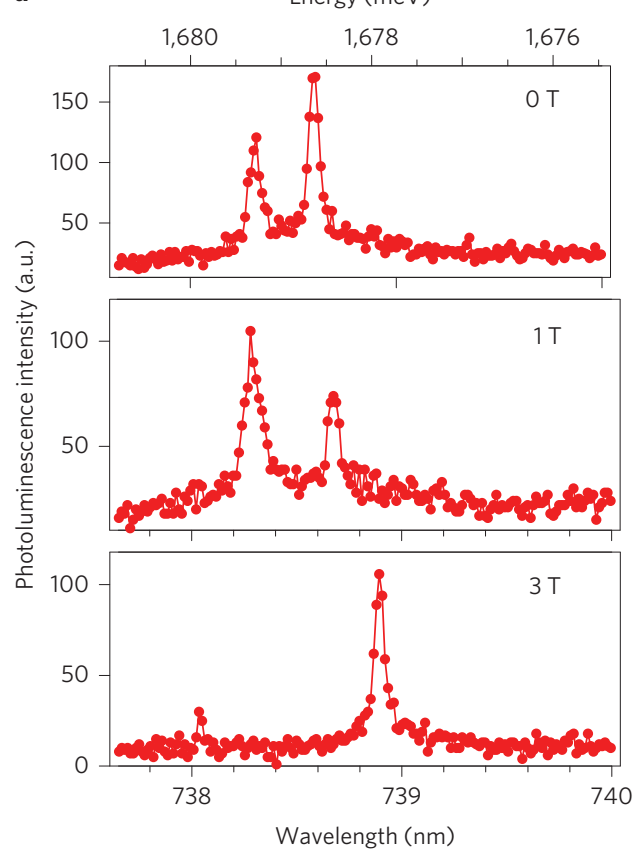

b
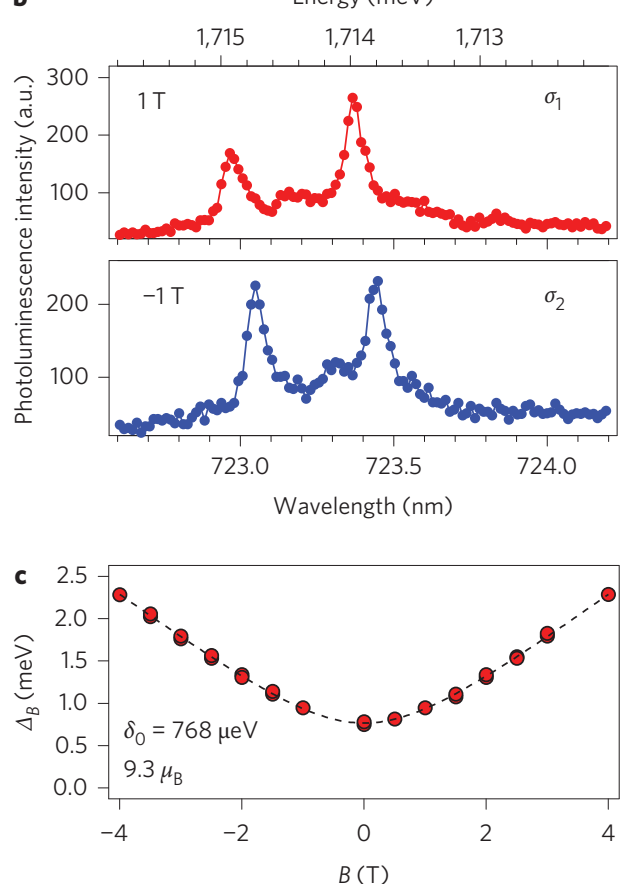

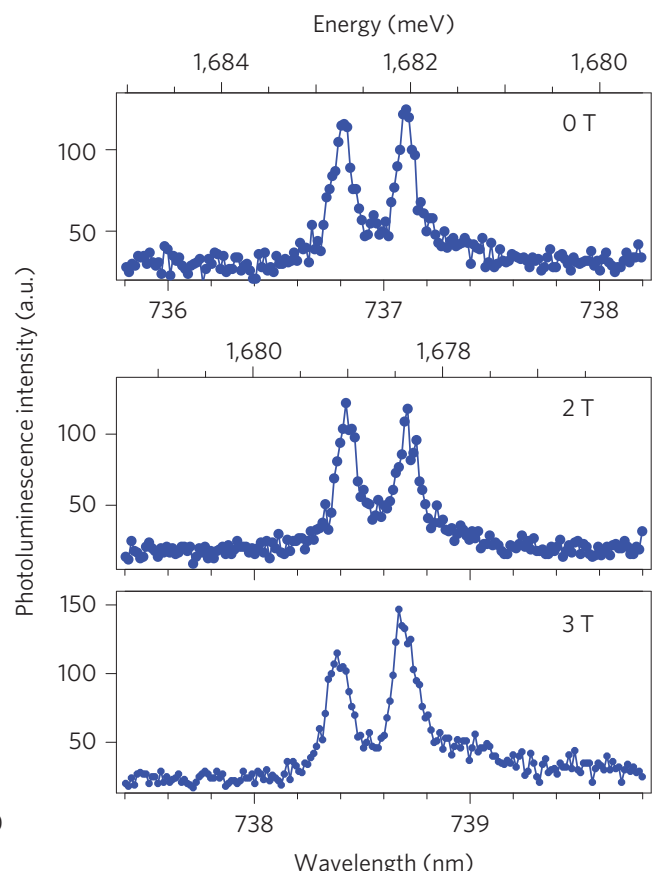

Wavelength (nm)

Energy (meV)
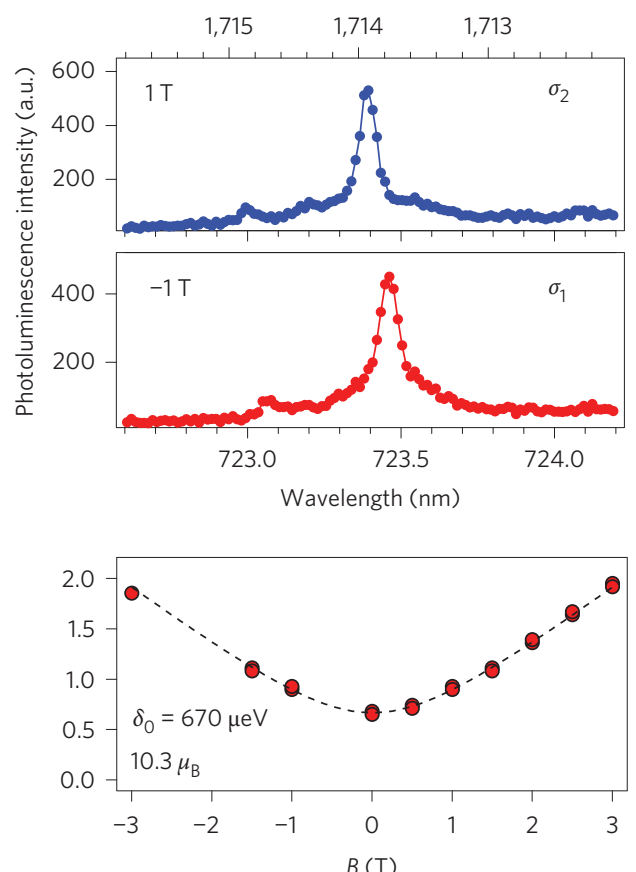

Figure 4 | Magnetic field dependence of quantum dot photoluminescence. a, Left: Polarization-resolved magnetic field ( $B$ ) dependence of photoluminescence from a quantum dot (QD1F2) in perpendicular B (Faraday geometry), showing splitting of the two peaks with increasing $B$. Photoluminescence is detected in a circularly-polarized basis, $\sigma_{1}$. Even though the high energy peak should be dominant at higher $B$ because of its emission helicity being that of the detection basis, efficient thermal relaxation causes the low energy peak to be stronger. In the opposite detection basis, $\sigma_{2}$ (b), the low energy peak is even stronger for $B>0$. Right: With $B$ parallel to the sample (Voigt geometry) no measurable splitting is observed. $\mathbf{b}$, Helicity of polarization of the two split peaks of quantum dot QD2F1, denoted by red and blue traces, switches sign on reversal of the direction of $B$. c, Extracted splitting between the two peaks in the Faraday geometry of QD2F1 (left) and QD1F2 (right) as a function of B is fitted with a hyperbolic function (see main text) to extract the magnetic moment and the zero-field exchange splitting $\left(\delta_{0}\right)$.

typical semiconductor quantum dots. In contrast, the exciton lifetime in $\mathrm{MoS}_{2}$ is reported to be $\sim 4$ ps (ref. 17) and is determined predominantly by non-radiative decay.

It is well known that zero-dimensional emitters in solids typically exhibit spectral diffusion, blinking and in some cases photobleaching. To determine whether $\mathrm{WSe}_{2}$ quantum dots also exhibit these properties (which would strongly limit their applications), we recorded the single photon detection events for QD1F1 using a single-photon-counting APD at a total photon counting rate of $\sim 13 \mathrm{kHz}$. We then used this data to obtain the waiting time distribution $W(\tau)$ for $\tau \geq 10 \mu$ s (refs 18,19). Figure 3a shows that $W(\tau)$ can be fitted by a single exponential 
where the decay time is given by $\tau_{\mathrm{det}}=(\Gamma \eta)^{-1} \approx 77 \mu$ s, in excellent agreement with the measured photon counting rate. Here, $\Gamma$ is the spontaneous emission rate and $\eta$ is the detection efficiency. This observation shows that there is no blinking for timescales longer than $\tau_{\text {det }}$. In the event of intensity intermittency or blinking, one expects the intensity to exhibit bright and dark states between which the system switches randomly. If this switching takes place on a timescale $\tau_{\text {blink }}>\tau_{\text {det }}$, then $W(\tau)$ should have an additional decay on a timescale determined by $\tau_{\text {blink }}{ }^{20}$. The absence of bunching on timescales $\leq 1 \mu \mathrm{s}$ in $g^{2}(\tau)$ measurements (not shown) in turn rules out blinking on such short timescales. Finally, we emphasize that blinking statistics in most quantum dots is known from earlier studies to be non-Poissonian, exhibiting power-law tails in the waiting time distribution ${ }^{21}$. In the absence of any such deviation from an exponential decay, we tentatively conclude that there is no significant blinking in the quantum dots studied here. However, we emphasize that this conclusion pertains to a rather small set of brightest localized emitters in the two flakes that exhibited sharp emission peaks.

We emphasize that there is no obvious degradation of photoluminescence intensity up to hundreds of microwatts of incident $\mathrm{HeNe}$ laser power, way above the linear response regime of quantum dots. The quantum dot photoluminescence also survived several cycles of warming to room temperature and cooling to $4.2 \mathrm{~K}$; these observations allow us to conclude that the quantum dots do not photo-bleach. However, spectral diffusion of the emission peak with an excursion range of a few hundred $\mu \mathrm{eV}$ is observed in almost all quantum dots but with varying strength. Most quantum dots exhibit small spectral diffusion with a range of $\pm 200 \mu \mathrm{eV}$, which cannot be resolved using a low-resolution spectrometer (Fig. 3b, left). On the other hand, we have observed that some quantum dots exhibit a large spectral diffusion of range $\pm 0.5 \mathrm{meV}$ (Fig. $3 \mathrm{~b}$, right). Such strong spectral diffusion, probably arising from charge fluctuations in the environment of the quantum dots, could last up to minutes before the peak energy is stable again.

Having established that the sharp emission peaks stem from optically active quantum dots, we focus on demonstrating that the quantum dots inherit their electronic properties, such as the valley degree of freedom, from $\mathrm{WSe}_{2}$. A priori, it is not clear whether the quantum dot confinement strongly mixes the two valleys, limiting the application of TMD quantum dots for valley-based information processing. In the following we provide evidence that the valley degree of freedom is robust in the TMD quantum dots studied here.

To demonstrate the existence of a valley degree of freedom for the observed quantum dots, we performed polarization-resolved magneto-optical spectroscopy with magnetic field ( $B$-field) both perpendicular (Faraday geometry) and parallel (Voigt geometry) to the flake. It has been reported recently that only in the presence of a magnetic field perpendicular to the TMD flake will exciton and trion emission peaks split into two circularly polarized peaks of opposite helicities due to the lifting of valley degeneracy ${ }^{16,22-24}$. This strongly anisotropic magnetic response was attributed to the two-dimensional electronic structure of TMDs. The magnetic field dependence of the photoluminescence obtained by a linearly polarized excitation laser and detected in the circularly-polarized basis in the Faraday geometry is shown in Fig. 4a (left). Two split peaks are observed, even at zero $B$-field, with the splitting increasing with increasing $B$-field. Most notably, no clear dependence of the splitting on $B$-field is observed in the Voigt geometry, just like in the case of the exciton and trion (Fig. $4 \mathrm{a}$, right). The split peaks are circularly polarized with opposite helicities at finite $B$-field, which reverse on reversing the direction of the $B$-field, confirming that the peaks arise from the same quantum dot (Fig. 4b). These observations strongly suggest that the quantum dots inherit their electronic structure from the host TMD and are very likely to be excitons trapped in shallow defect or impurity potentials. Our findings also lend support to the recent prediction that valley hybridization is absent in TMD quantum dots, preserving the valley physics of two-dimensional bulk ${ }^{25}$.

The observed zero-field splitting of $\sim 700 \mu \mathrm{eV}$ possibly originates from an electron-hole exchange interaction of a neutral exciton trapped in an asymmetric confining potential, as is commonly observed in self-assembled InAs/GaAs quantum dots ${ }^{26}$. It could also arise if the exciton is bound to an ionized donor, as no electron-hole exchange interaction is present when the exciton is bound to a neutral donor with a spin-1/2 ground state. It is noteworthy that this zero-field splitting in TMD quantum dots is almost 50 times larger than that in InAs/GaAs self-assembled quantum dots ${ }^{26}$, consistent with the strong Coulomb interactions in monolayer TMDs.

The splitting between the two peaks is plotted against $B$-field in Fig. $4 \mathrm{c}$ and fit to the hyperbolic dispersion

$$
E(B)=\sqrt{\mu^{2} B^{2}+\delta_{0}^{2}}
$$

expected for exchange-mixed circularly-polarized resonances. We obtain $\left|\delta_{0}\right|$ between 650 and $850 \mu \mathrm{eV}$ and $\mu$ between 7.5 and $10.9 \mu_{\mathrm{B}}$ (Bohr magneton) after fitting splitting data for several different quantum dots in two different flakes (Supplementary Fig. 2). This measured magnetic moment of the quantum dot exciton is significantly larger than that of a free exciton $\left(\sim 4 \mu_{\mathrm{B}}\right)$ and a trion $\left(\sim 5.5-6 \mu_{\mathrm{B}}\right)$ in $\mathrm{WSe}_{2}$ (ref. 16). The origin of the remarkably large anomalous $g$-factor in the TMD quantum dots studied here remains elusive.

We also performed photoluminescence excitation spectroscopy and observed an overall enhancement in photoluminescence for most quantum dots when the laser was tuned close to the free excitonic resonance (Supplementary Fig. 3).

An earlier study on defect-activated photoluminescence in TMDs concluded that the impurity band emission arises from excitons trapped in anion vacancies ${ }^{15}$, which could also be the origin of the quantum dots studied here. In addition, recent high-resolution transmission electron microscopy studies have revealed the presence of line defects and island-like domains within a single $\mathrm{MoS}_{2}$ layer27. We observed strong emission from individual quantum dots in two flakes, whereas most other monolayer flakes (approximately 10) showed behaviour similar to flake 0 , presumably due to a much higher density of quantum dots leading to the formation of an impurity band.

Quantum dots in TMDs are likely to have very favourable properties for applications in quantum information processing ${ }^{28,29}$. First and foremost, a qubit defined using the two lowest energy states of an excess quantum-dot electron could be classified as a spin-valley qubit. Because the spin and valley degrees of freedom are strongly correlated due to a large spin-orbit interaction, any decoherence mechanism that couples only to spin or to valley will remain ineffective. Magnetic impurities are likely to be the dominant source of pure decoherence. Unlike GaAs, however, the dominant isotopes of most TMDs are nuclear-spin free, rendering material-qualityindependent hyperfine decoherence to be weak. The ability to position defects that trap excitons within a monolayer would provide a major boost to TMD quantum dot research.

Note added in proof: After submission of the manuscript, we became aware of similar results by three other groups ${ }^{30-32}$.

\section{Methods}

The samples studied in the present experiments were obtained by mechanical exfoliation from $\mathrm{WSe}_{2}$ synthetic crystals onto heavily doped silicon substrates with a $285 \mathrm{~nm} \mathrm{SiO}_{2}$ layer on top ${ }^{33,34}$. Monolayer flakes were identified from their optical contrast. Polarization-resolved photoluminescence and resonant white-light reflection spectroscopy were performed using a home-built confocal microscope set-up 
placed in a liquid-helium bath cryostat. The sample temperature was $4.2 \mathrm{~K}$ and the excitation source was a HeNe laser at $632.8 \mathrm{~nm}$ or a tunable continuous-wave Ti: sapphire laser. Magnetic fields in the range $\pm 8.4 \mathrm{~T}$ were applied both parallel and perpendicular to the plane of the sample. Polarization control of excitation and photoluminescence was performed using a liquid-crystal retarder calibrated for halfand quarter-wavelength retardance at the emission wavelengths. Photoluminescence lifetime measurements were performed using a Ti:sapphire laser operating in pulsed mode with an $\sim 5$ ps pulse width at a repetition rate of $76 \mathrm{MHz}$. For lifetime and photon correlations measurements, photoluminescence was spectrally filtered around the quantum dot wavelength using a transmission grating before sending the signal to APDs with a timing resolution of $\sim 350 \mathrm{ps}$. Second-order photon correlation measurements were performed using two silicon APDs in a standard HBT set-up and analysed using a two-channel time-correlated single-photoncounting module (PicoHarp 300). The waiting time distribution was obtained using a single APD (dead time of $\sim 200 \mathrm{~ns}$ ) to record individual photon detection events with their arrival time in time-tagged time-resolved (TTTR) modes of PicoHarp 300.

\section{Received 11 November 2014; accepted 4 March 2015; published online 4 May 2015}

\section{References}

1. DiVincenzo, D. P. The physical implementation of quantum computation. Fortschr. Phys. 48, 771-783 (2000).

2. Imamoğlu, A. et al. Quantum information processing using quantum dot spins and cavity QED. Phys. Rev. Lett. 83, 4204-4207 (1999).

3. Gao, W-B., Fallahi, P., Togan, E., Miguel-Sanchez, J. \& Imamoğlu, A. Observation of entanglement between a quantum dot spin and a single photon. Nature 491, 426-430 (2012).

4. Xu, X., Wang, Y., Di, X. \& Heinz, T. F. Spins and pseudospins in layered transition metal dichalcogenides. Nature Phys. 10, 343-350 (2014).

5. Lee, C-H. et al. Atomically thin $\mathrm{p}-\mathrm{n}$ junctions with van der Waals heterointerfaces. Nature Nanotech. 9, 676-681 (2014).

6. Xiao, D., Liu, G-B., Feng, W., Xu, X. \& Yao, W. Coupled spin and valley physics in monolayers of $\mathrm{MoS}_{2}$ and other group-VI dichalcogenides. Phys. Rev. Lett. 108, 196802 (2012)

7. Dousse, A. et al. Ultrabright source of entangled photon pairs. Nature 466, 217-220 (2010).

8. Berezovsky, J., Mikkelsen, M. H., Stoltz, N. G., Coldren, L. A. \& Awschalom, D. D. Picosecond coherent optical manipulation of a single electron spin in a quantum dot. Science 320, 349-352 (2008).

9. Press, D., Ladd, T. D., Zhang, B. \& Yamamoto, Y. Complete quantum control of a single quantum dot spin using ultrafast optical pulses. Nature 456, 218-221 (2008)

10. De Greve, K. et al. Quantum-dot spin-photon entanglement via frequency downconversion to telecom wavelength. Nature 491, 421-425 (2012).

11. Elzerman, J. M. et al. Single-shot read-out of an individual electron spin in a quantum dot. Nature 430, 431-435 (2004).

12. Petta, J. R. et al. Coherent manipulation of coupled electron spins in semiconductor quantum dots. Science 309, 2180-2184 (2005).

13. Wilson-Rae, I., Galland, C., Zwerger, W. \& Imamoglu, A. Exciton-assisted optomechanics with suspended carbon nanotubes. New J. Phys. 14, 115003 (2012).

14. Jones, A. M. et al. Optical generation of excitonic valley coherence in monolayer WSe ${ }_{2}$. Nature Nanotech. 8, 896-869 (2013).

15. Tongay, S. et al. Defects activated photoluminescence in two-dimensional semiconductors: interplay between bound, charged, and free excitons. Sci. Rep. 3, 2657 (2013).

16. Srivastava, A. et al. Valley Zeeman effect in elementary optical excitations of a monolayer $\mathrm{WSe}_{2}$. Nature Phys. 11, 141-147 (2015).
17. Lagarde, D. et al. Carrier and polarization dynamics in monolayer $\mathrm{MoS}_{2}$. Phys. Rev. Lett. 112, 047401 (2014).

18. Reynaud, S. La fluorescence de résonance: Étude par la méthode de l'atome habillé. Ann. Phys. (Paris) 8, 315 (1983).

19. Lounis, B., Bechtel, H. A., Gerion, D., Alivisatos, P. \& Moerner, W. E. Photon antibunching in single CdSe/ZnS quantum dot fluorescence. Chem. Phys. Lett. 329, 399-404 (2000).

20. Delteil, A., Gao, W-B., Fallahi, P., Miguel-Sanchez, J. \& Imamoğlu, A. Observation of quantum jumps of a single quantum dot spin using submicrosecond single-shot optical readout. Phys. Rev. Lett. 112, 116802 (2014).

21. Frantsuzov, P., Kuno, M., Janko, B. \& Marcus, R. A. Universal emission intermittency in quantum dots, nanorods and nanowires. Nature Phys. 4, 519-522 (2008).

22. MacNeill, D. et al. Breaking of valley degeneracy by magnetic field in monolayer $\mathrm{MoSe}_{2}$. Phys. Rev. Lett. 114, 037401 (2015).

23. Aivazian, G. et al. Magnetic control of valley pseudospin in monolayer $\mathrm{WSe}_{2}$. Nature Phys. 11, 148-152 (2015).

24. Li, Y. et al. Valley splitting and polarization by the Zeeman effect in monolayer $\mathrm{MoSe}_{2}$. Phys. Rev. Lett. 113, 266804 (2014).

25. Liu, G-B., Pang, H., Yao, Y. \& Yao, W. Intervalley coupling by quantum dot confinement potentials in monolayer transition metal dichalcogenides. New J. Phys. 16, 105011 (2014).

26. Gammon, D., Snow, E. S., Shanabrook, B. V., Katzer, D. S. \& Park, D. Homogeneous linewidths in the optical spectrum of a single gallium arsenide quantum dot. Science 273, 87-90 (1996).

27. Enyashin, A. N., Bar-Sadan, M., Houben, L. \& Seifert, G. Line defects in molybdenum disulfide layers. J. Phys. Chem. C 117, 10842 (2013).

28. Klinovaja, J. \& Loss, D. Spintronics in $\mathrm{MoS}_{2}$ monolayer quantum wires. Phys. Rev. B 88, 075404 (2013).

29. Kormányos, A., Zólyomi, V., Drummond, N. D. \& Burkard, G. Spin-orbit coupling, quantum dots, and qubits in monolayer transition metal dichalcogenides. Phys. Rev. X 4, 011034 (2014).

30. He, Y.-M. et al. Single quantum emitters in monolayer semiconductors. Nature Nanotech. http://dx.doi.org/10.1038/nnano.2015.75 (2015).

31. Koperski, M. et al. Single photon emitters in exfoliated $\mathrm{WSe}_{2}$ structures. Nature Nanotech. http://dx.doi.org/10.1038/nnano.2015.67 (2015).

32. Chakraborty, C. et al. Voltage-controlled quantum light from an atomically thin semiconductor. Nature Nanotech. http://dx.doi.org/10.1038/nnano.2015.79 (2015).

33. Novoselov, K. S. et al. Two-dimensional atomic crystals. Proc. Natl Acad. Sci. USA 102, 10451-10453 (2005).

34. Radisavljevic, B., Radenovic, A., Brivio, J., Giacometti, V. \& Kis, A. Single-layer $\mathrm{MoS}_{2}$ transistors. Nature Nanotech. 6, 147-150 (2011).

\section{Acknowledgements}

This work is supported by NCCR Quantum Science and Technology (NCCR QSIT), a research instrument of the Swiss National Science Foundation (SNSF).

\section{Author contributions}

A.S. and M.S. carried out the optical measurements. A.V.A., D.S.L. and A.K. prepared the samples. A.S. and A.I. supervised the project and analysed the experimental data. All authors contributed extensively to this work.

\section{Additional information}

Supplementary information is available in the online version of the paper. Reprints and permissions information is available online at www.nature.com/reprints. Correspondence and requests for materials should be addressed to A.S. and A.I.

\section{Competing financial interests}

The authors declare no competing financial interests. 